\title{
Possible Factors Associated with Low Case Fatality Rate of COVID-19 in Kurdistan Region, Iraq
}

\author{
Nawfal R Hussein ${ }^{1, *}$ \\ ${ }^{1}$ Department of Biomolecular Sciences, College of Medicine, University of Zakho, Zakho, Iraq \\ "Corresponding author: Department of Biomolecular Sciences, College of Medicine, University of Zakho, Zakho, Iraq. Tel: +96-4627649807, Email: nawfal.hussein@yahoo.com
} Received 2020 April 04; Accepted 2020 April 11.

Keywords: Kurdistan, Iraq, Coronavirus, COVID-19

\section{Dear Editor,}

The first case of COVID-19 was diagnosed on the 1st of March 2020 in Kurdistan Region of Iraq. Since then, 171 cases were diagnosed in the region, amongst these 82/171 (47.95\%) cases were female (1). The age of diagnosed patients ranged from 2 to 80 years old. 128/171 (74.9\%) were diagnosed through routine testing at the end of quarantine period and were symptoms free (1). Amongst our patients, 34/171 (19.9\%) patients presented with symptoms, $61 / 171$ (35.7\%) recovered and 2/171 passed away giving a case fatality rate of $1.17 \%$ (1). Careful examination of data from the region showed that the majority of our patients were asymptomatic and the case fatality rate was very low. Besides, despite the limited resources in the region, the cure rate was high.

In a recent study compared the morbidity and mortality among different countries, it was found that countries that implement universal policies of BCG vaccination, such as Japan, maintained low case fatality, while countries without BCG universal vaccination program such as Italy, England and Spain have been more severely affected with high case fatality rate (2). In Kurdistan Region, BCG vaccine program started in late 1950 s with a good vaccination coverage rate. Though the correlation between BCG and COVID-19 vaccine yet to be determined, it may explain the low case fatality rate and high cure rate in our region. In addition, studies analyzing 103 genomes of SARS CoV-2 showed that genomes were genotyped into two types; evolutionarily older and less aggressive $S$ (the ancestor) type and aggressive and quickly spread L type (3). Moreover, continuous evolution of the virus by successive mutations and recombination processes has been reported (4). The low mortality and morbidity in Kurdistan region and other countries could be explained partially by a possible variation in the genetic make up of the virus. Therefore; urgent comprehensive research is needed to investigate the mutagenesis of the virus in the region.

Previous studies suggest a crucial role of immune response in the pathogenesis of coronavirus (5). Besides, it was previously shown that HLA gene polymorphisms influence the outcome of patients with SARS-CoV virus (5). Moreover, it has been shown recently that ACE2 could be the host receptor for the novel SARS-CoV-2. Additionally, it was found that ACE2 gene polymorphism may be associated with higher expression levels of ACE2 in some populations (6). Hence, the difference in mortality and morbidity rates amongst different countries may be explained by genetic susceptibility. Further studies are needed to investigate human gene polymorphisms and its role in disease pathogenesis.

To conclude, the low fatality rate in the region can be explained partially by the high coverage rate of BCG vaccine, different genotypes of the virus and/or genetic makeup of the patients. Further studies are required to investigate these factors and confirm their roles in disease pathogenesis.

\section{Footnotes}

Conflict of Interests: The author has nothing to declare. Funding/Support: None declared by author.

\section{References}

1. MoH. Latest Information about Coronavirus (COVID-19). MoH; 2020, [cited 3/4/2020]. Available from: https://gov.krd/coronavirus-en/.

2. Miller A, Reandelar MJ, Fasciglione K, Roumenova V, Li Y, Otazu GH. Correlation between universal BCG vaccination policy and reduced morbidity and mortality for COVID-19: an epidemiological study. medRxiv. 2020:2020.03.24.20042937. doi: 10.1101/2020.03.24.20042937. 
3. Tang X, Wu C, Li X, Song Y, Yao X, Wu X, et al. On the origin and continuing evolution of SARS-CoV-2. National Science Review. 2020. doi: 10.1093/nsr/nwaa036.

4. Yi H. 2019 novel coronavirus is undergoing active recombination. Clinical Infectious Diseases. 2020. doi: 10.1093/cid/ciaa219.

5. Yuan FF, Velickovic Z, Ashton LJ, Dyer WB, Geczy AF, Dunckley $\mathrm{H}$, et al. Influence of HLA gene polymorphisms on susceptibil- ity and outcome post infection with the SARS-CoV virus. Virologica Sinica. 2014;29(2):128-30. doi: 10.1007/s12250-014-3398-x. [PubMed 24643938].

6. Cao Y, Li L, Feng Z, Wan S, Huang P, Sun X, et al. Comparative genetic analysis of the novel coronavirus (2019-nCoV/SARS-CoV-2) receptor ACE2 in different populations. Cell Discovery. 2020;6(1):11. doi: 10.1038/s41421-020-0147-1. 